\title{
Towards stable monolithic perovskite/silicon tandem photovoltaics: A six-month outdoor performance study in a hot climate
}

Michele De Bastiani, * Emmanuel Van Kerschaver, Quentin Jeangros, Atteq Ur Rehman, Erkan Aydin, Furkan H. Isikgor, Alessandro J. Mirabelli, Maxime Babics, Jiang Liu, Shynggys Zhumagali, Esma Ugur, George T. Harrison, Thomas G. Allen, Bin Chen, Yi Hou, Semen Shikin, Edward H. Sargent, Christophe Ballif, Michael F. Salvador, and Stefaan De Wolf*

\section{Supporting Information}

\section{Experimental part:}

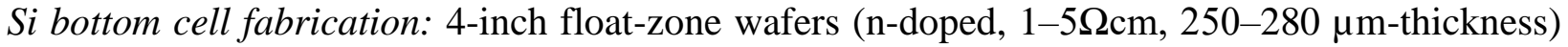
were used to fabricate silicon heterojunction (SHJ) bottom cells. Firstly, an alkaline solution was used to prepare a double-side texturing, followed by RCA-I and RCA-2 cleaning. To remove the native oxide layer, the wafers were dipped in 5\% Hydrofluoric acid solution. The amorphous silicon (intrinsic, $\mathrm{n}$ and p) layers were deposited in an Indeotec Octopus II - Plasma enhanced chemical vapor deposition (PECVD) chamber with silane, hydrogen, phosphine and trimethylboron as input gases. In the subsequent steps nc-Si:H(n) recombination junction and a$\mathrm{Si}: \mathrm{H}(\mathrm{p})$ is formed by deposition of $\mathrm{n}$ - and $\mathrm{p}$-doped silicon films utilizing high hydrogen: silicon gas flow ratios. Recombination junction ITO $(25 \mathrm{~nm})$, rear ITO $(150 \mathrm{~nm})$ and back Ag electrode $(250 \mathrm{~nm})$ were deposited in physical vapour deposition (PVD) part of the Octopus cluster. All these processes were performed at temperatures $\leq 200^{\circ} \mathrm{C}$.

Tandem fabrication: On the recombination junction, $17 \mathrm{~nm}$ of $\mathrm{NiOx}$ were sputtered in an Angstrom EvoVac deposition chamber and subsequently washed with bromobenzoic acid. A perovskite solution (1.7M) was prepared by mixing $36.4 \mathrm{mg}$ of CsI (Alfa Aesar), $44.8 \mathrm{mg}$ of methylammonium bromide (Greatcell), $389 \mathrm{mg}$ of formamidinium iodide (Greatcell), $1198 \mathrm{mg}$ of $\mathrm{PbI}_{2}$ (Alfa Aesar), $73.4 \mathrm{mg}$ of $\mathrm{PbBr}_{2}$ (Sigma Aldrich) with 4:1 DMF:DMSO. The solution was spin coated on the bottom cells at $2000 \mathrm{rpm}$ for 60 seconds; 11 seconds before the end of the process, the spin speed increased to $5000 \mathrm{rpm}$ and anisole was dripped as solvent quencher. $20 \mathrm{~nm}$ of $\mathrm{C}_{60}$ were thermally evaporated (Angstrom EvoVac) on top of the perovskite. On top of the $\mathrm{C}_{60}$ layer, SnOx was deposited as buffer layer via atomic layer deposition (Picosun). Indium Zinc Oxide was sputtered as transparent top electrode covered by an evaporated grid of $350 \mathrm{~nm}$ of silver as contact (both, Angstrom EvoVac). Finally, $90 \mathrm{~nm}$ of $\mathrm{MgF}_{2}$ were evaporated as antireflective coating.

Encapsulation and test-field measurements: For the encapsulation, silver tabbing wires were connected to the front and rear contact using silver paste and vacuum annealed at $120{ }^{\circ} \mathrm{C}$ for 10 min. Encapsulation with butyl rubber as edge sealant (Quanex, SET LP03) was performed in a 
Ecoprogetti Ecolam 5. After the encapsulation, rigid masks are firmly attached to the front and rear glass to define the aperture area of the devices. To prevent moisture entering through the tabs, we applied a layer of HelioSeal PVS 101 (HB fuller). In the test field, a $J(V)$-curve is recorded every ten minutes. In between $J(V)$ measurements, the device was kept under open circuit condition. The latter has been shown to be the most severe testing conditions, compared to keeping the device under short circuit or maximum power point (MPP) tracking. ${ }^{1}$ A pyranometer positioned in plane with the devices recorded the intensity of the solar irradiation.

Characterization: A tandem cell aged in the field for 6 months was assessed by STEM EDX. In that regard, a lamella of a few $\mu \mathrm{m}^{2}$ was extracted at the position of a grid finger using the FIB liftoff method and then thinned down to electron transparency using a final gallium beam voltage of $5 \mathrm{kV}$. The sample was then exposed to air for 1 minute and transferred to a TFS Osiris transmission electron microscope equipped with 4 silicon drift detectors for fast EDX mapping. The microscope was operated at $200 \mathrm{kV}$ with a STEM beam current of $250 \mathrm{pA}$. The PL spectra of the encapsulated perovskite/silicon tandem device were collected using a hyperspectral imaging system coupled to a microscope with $2 \mathrm{~nm}$ spectral resolution (Photon etc. IMA). The sample was excited with 532 $\mathrm{nm}$ laser by focusing close to the Ag finger electrodes on the device immediately after the light soaking. Further, we performed the same measurement with certain time intervals to observe the recovery of the PL for the preconditioned device.

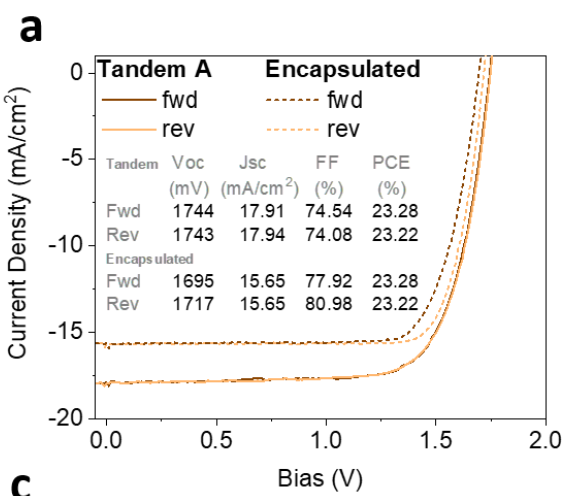

b
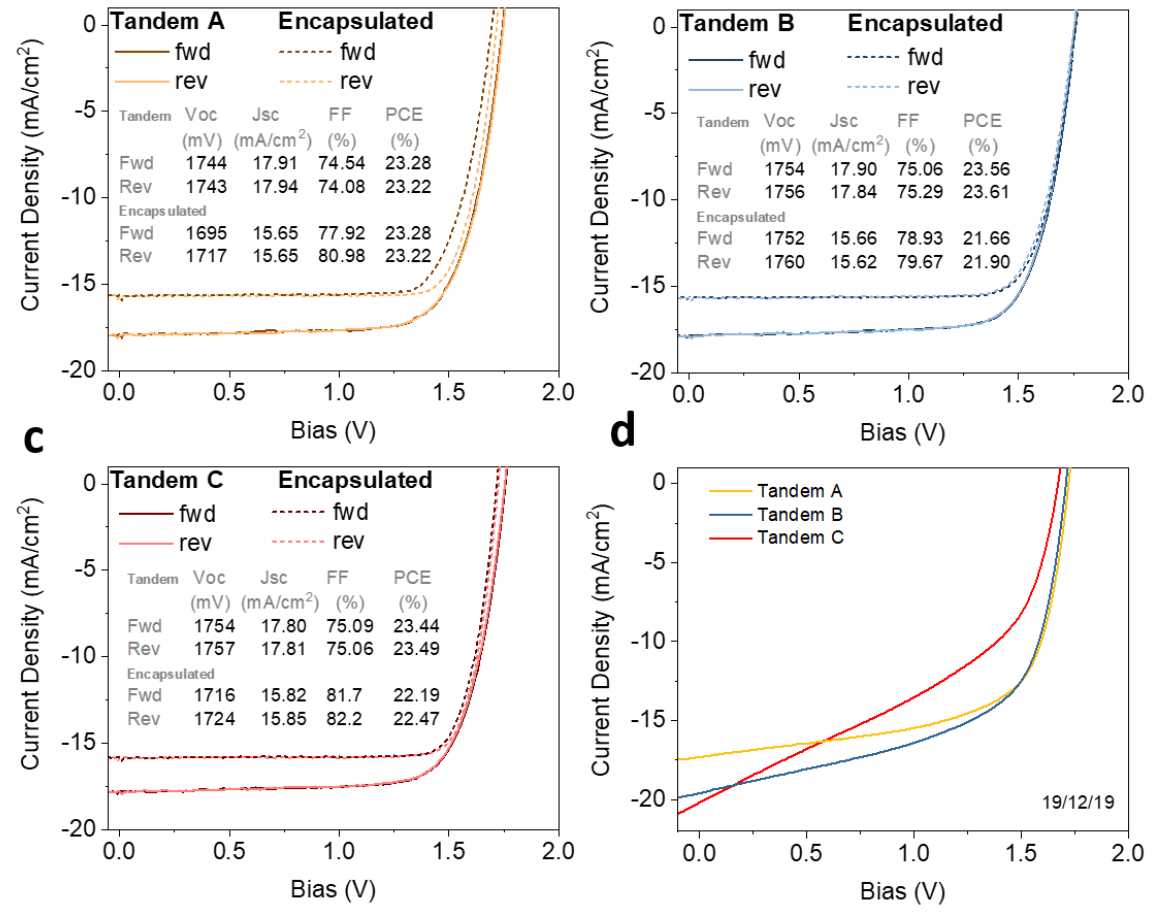
Figure S1. $J(V)$-curves of the bifacial tandems measured before and after encapsulation under standard condition and in the first day in the test-field. (a-c) Sample A, B, and C are intended to work in bifacial configuration, therefore when measured under standard condition (with 1-sun irradiation from the front) the devices are current-limited. The active area of the devices is defined by an aperture mask $\left(1.03 \mathrm{~cm}^{2}\right)$. After the encapsulation the $J \mathrm{sc}$ is reduced for two reasons: $i$ Front reflection induced by the front glass; $i i$ mismatch in the refracting index: air (outside)/glass/air (inside)/tandem. (d) $J(V)$ curves of sample A, B, and C the first day in the testfield at noon.

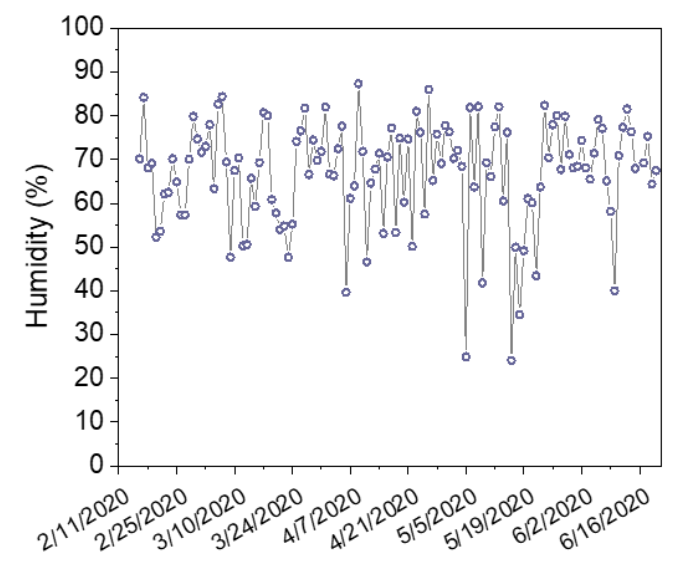

Figure S2. Environmental humidity recorded in our test-field site trough a weather station during the experiment. 

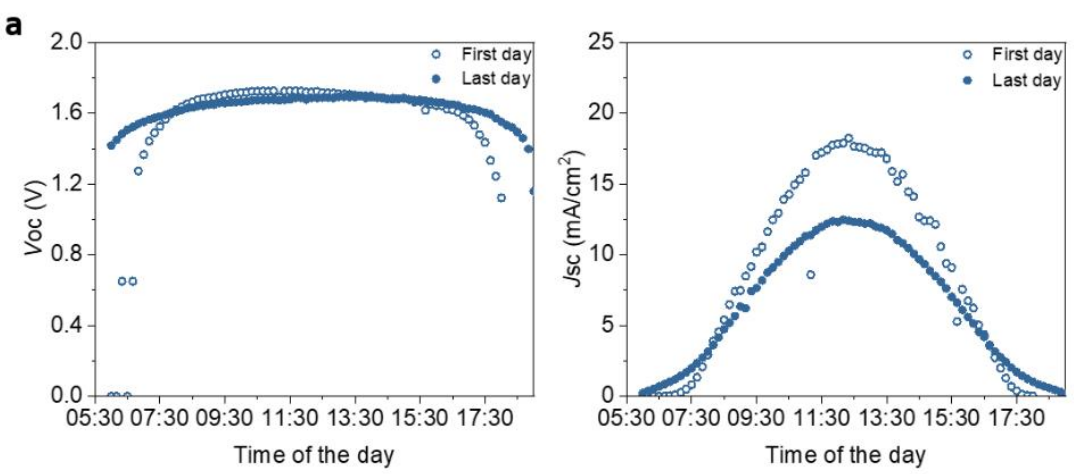

b
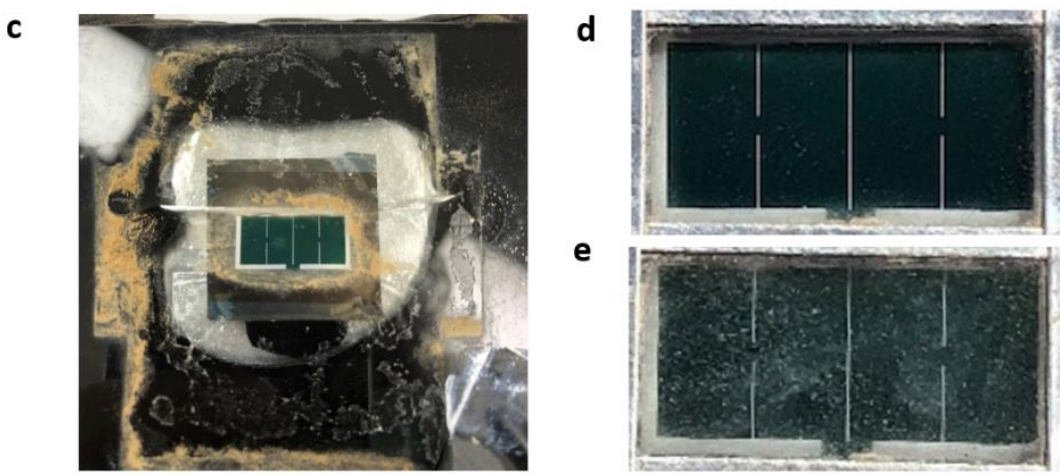

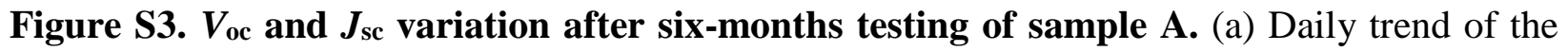
Voc over the first (open circles) and last (full circles) days in the test-field. (b) Daily trend of the Jsc over the first (open circles) and last (full circles) days in the test-field. (c) Front side of the encapsulated tandem after six months in the test-field, after removing the shadow masks at the front and rear. Dust accumulates also under the shadow mask. (d) Active area at the beginning of the test and after six months of testing (e).
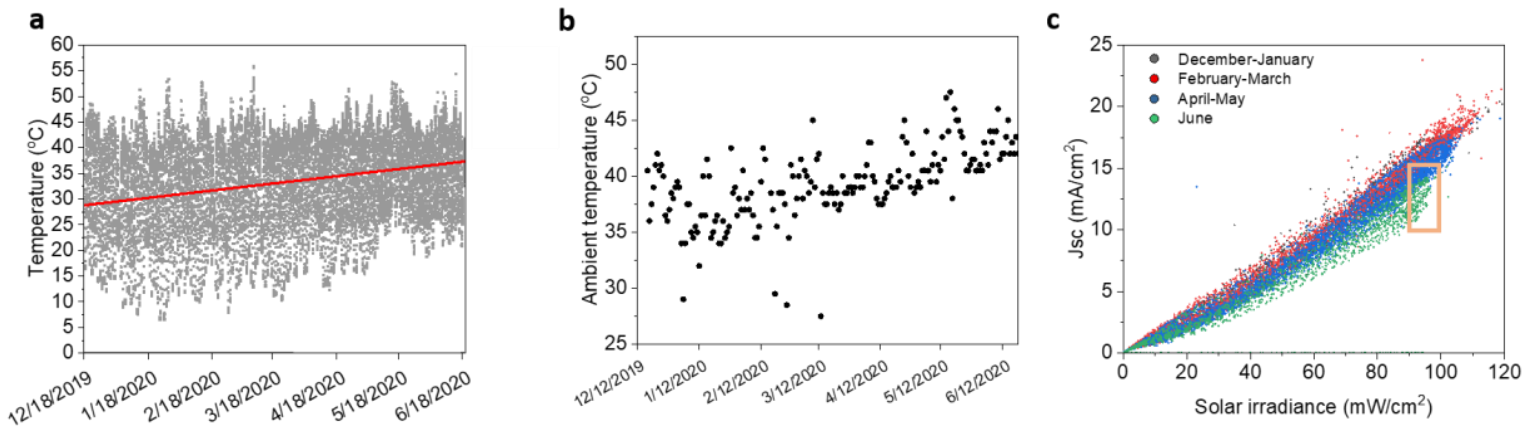
Figure S4. Temperature monitoring of a tandem device, ambient temperature, and current trend. During the test-field experiment, we recorded the temperature of an encapsulated tandem device with a thermocouple placed on the rear glass (a). The glass encapsulation $\left(49 \mathrm{~cm}^{2}\right)$ acts as temperature sink, reducing the actual temperature of the tandem. The linear fit highlights the increasing of the temperature going from the cold to hot season. The slope of the fitting is: 0.048 \pm 0.001 degree/day. (b) Ambient temperature measured in our test-field site with a weather station. (c) Jsc plotted versus the solar irradiance for the six-month experiment. The yellow rectangle highlights the current loss during the last month of experiment.

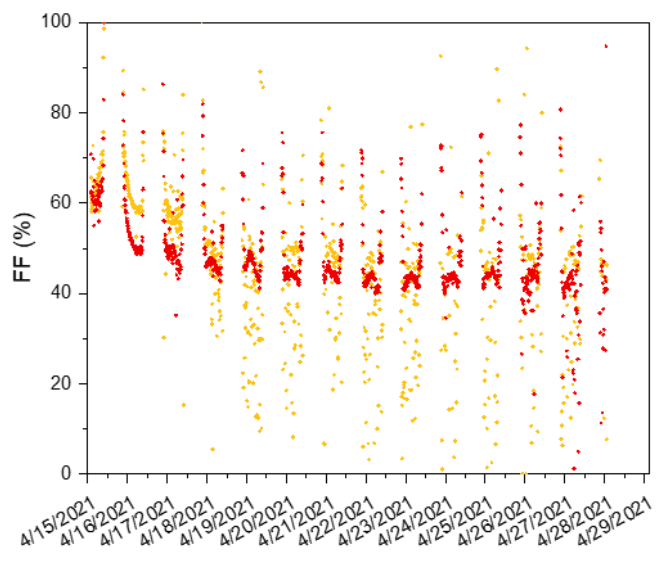

Figure S5. FF variation on single junction perovskite solar cells in the test-field. The two devices (red and yellow) share a similar device configuration with the bifacial tandems, with the rear electrode composed of $\mathrm{C} 60 / \mathrm{SnOx} / \mathrm{IZO} / \mathrm{Ag}$. The devices are encapsulated between two glasses similarly to the tandem, with butyl rubber as edge sealant. However, due to the superstrate configuration, light impinges on the HTL side and the rear Ag contact is evaporated full-area. 
a
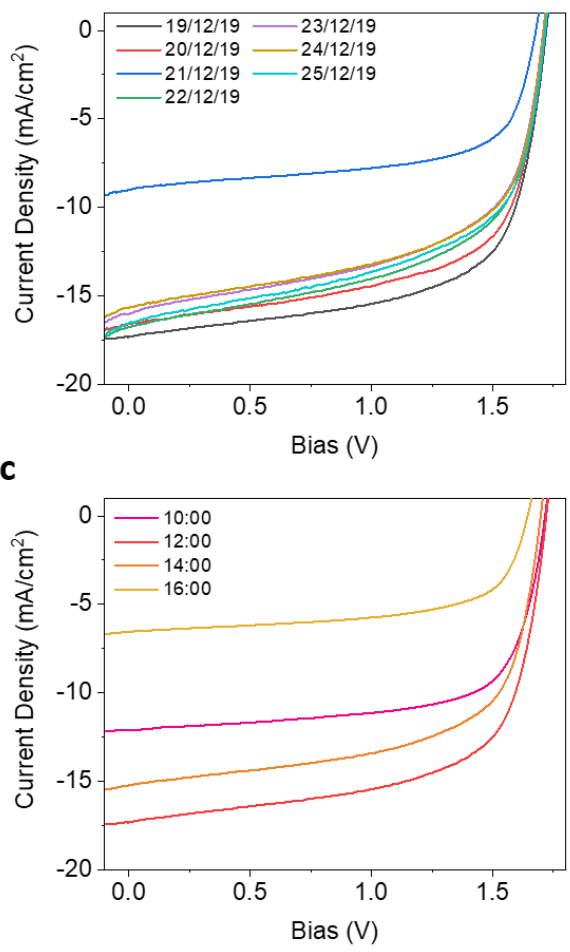
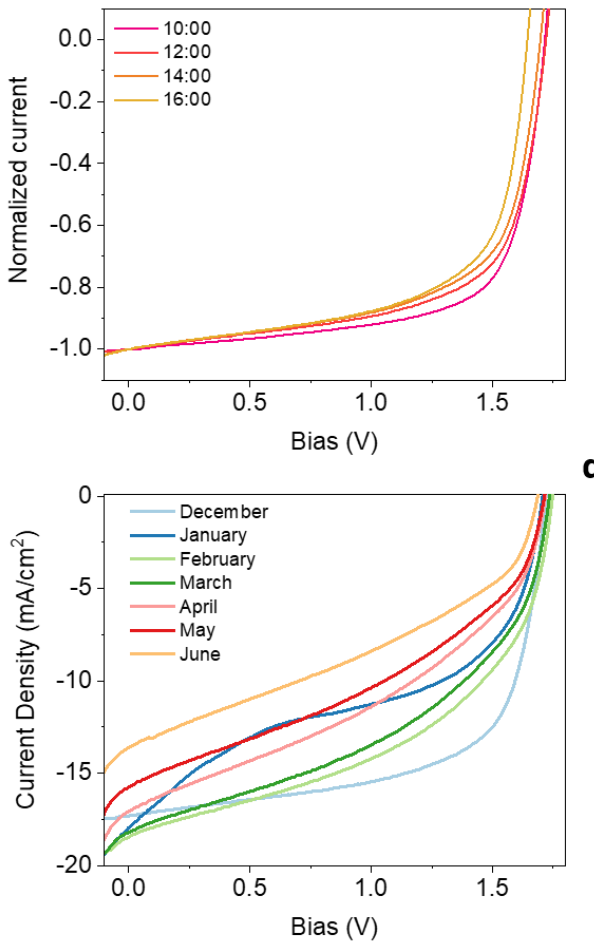

Figure S6. Analysis of the $J(V)$ curves of sample A recorded during the outdoor experiment.

(a) $J(V)$ curves recorded the first week (from $19 / 12 / 2019$ to $25 / 12 / 2019$ ) at noon. The $21^{\text {st }}$ December was characterized by low irradiance. Even during the first week we notice a reduction of the $F F$, particularly in a reduction of the $\mathrm{R}_{\text {shunt }}$. Variation in the Jsc are due to daily variation of the irradiance. (b-c) $J(V)$ curves recorded the first day (19/12/2019) in the morning, at noon, in the afternoon, and evening. The curves are normalized for figure $b$ to compare the $F F$. During the first day the device shows a reduction of the $F F$ compared to the lab measurements. This is due to two factors: $i$ ) presence of the albedo, which increase the current output to the matching condition between the two sub-cells, at the same time lowering the FF; ii) effect of temperature. (d) $J(V)$ curves recorded for each month, at noon, in days of high irradiance. Here, it is clear that the reduction of the $F F$ is linked to a gradually shunting of the device over time. 
a
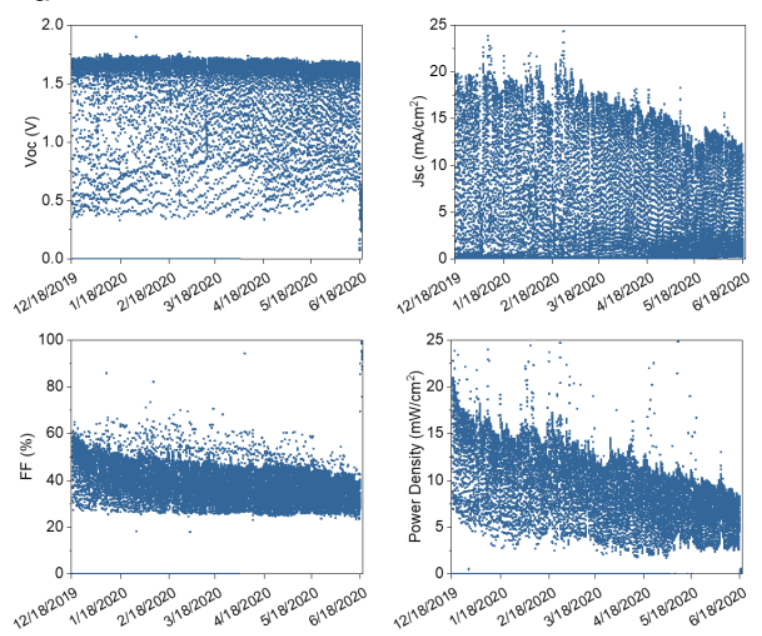

C
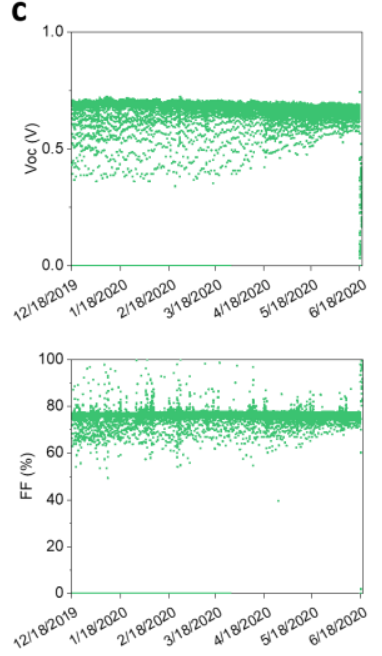

b
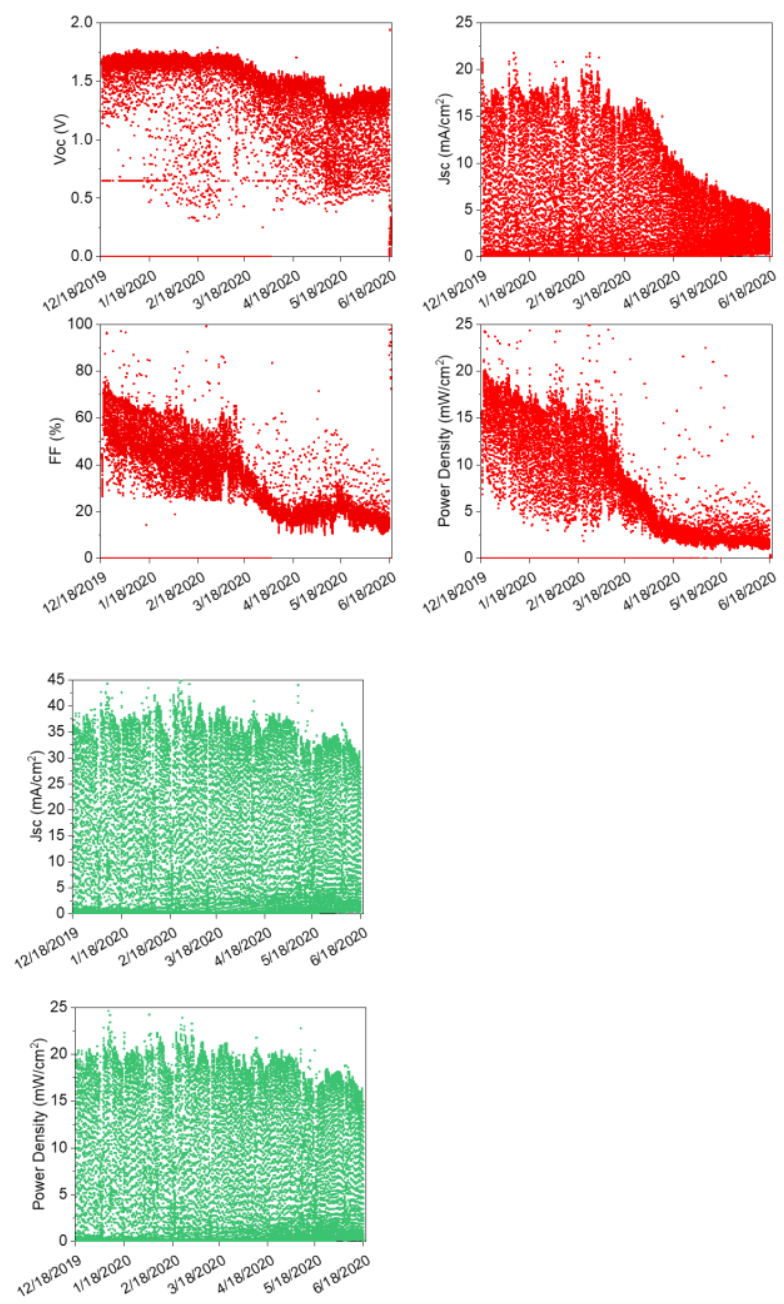

Figure S7. Test-field performances of the tandems and the silicon heterojunction reference over six months. Figures of merit for sample b (a), sample c (b),SHJ (c).

\section{Supplementary note: laboratory setup for lab experiments.}

After six-months of outdoor testing we removed sample A from the test-field and immediately performed a series of test in a lab-controlled environment to simulate the behavior of the sample as it was outdoor in the field. Several of these experiments included temperature controlled $J(V)$ measurements and for this reason we could not benefit from a bifacial setup. For a bifacial experiment, two lamps are required (front irradiation and albedo) with a transparent window to 
allow illumination on both sides. In this configuration, it is not possible to guarantee a precise control of the device temperature. Alternatively, the device can be placed between two mirrors, forming a 45 degree angle with incident light. In this configuration, the sample is free-standing within the mirrors. Once more, temperature control cannot be performed precisely. For these reasons, we opted for a precise control of the temperature, over a bifacial setup. It follows that the FF recorded in the lab-controlled experiment is higher than what recorded in the test-field, as the tandem is silicon-limited (similarly to what reported in Fig. S1).

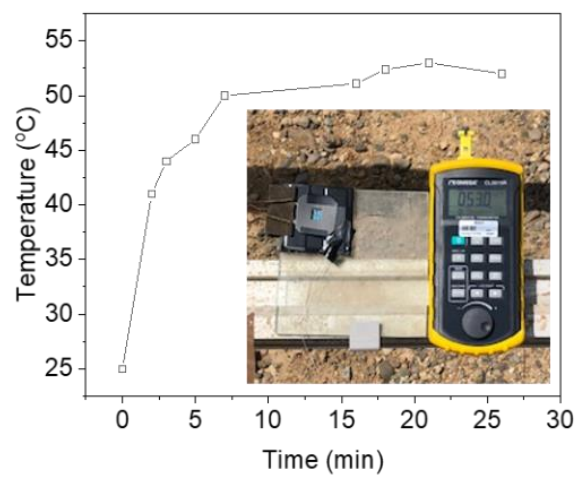

Figure S8. Rising of the temperature for a device in the outdoor. We probe the rising of the temperature of a freshly prepared tandem with a thermocouple placed at the front, under the shadow mask, to probe the highest temperature achieved by the glass packaging (see photograph in the inset). 


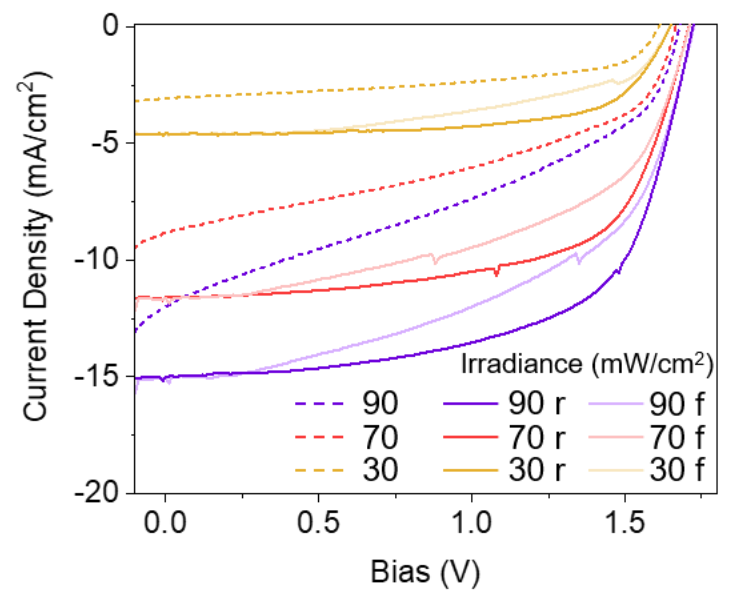

Figure S9. Comparison between $\mathbf{J}(\mathbf{V})$ curves at different light intensities. The $J(V)$ curves obtained from the lab experiment of Figure $3 \mathrm{~b}$ (full line) are reported both for forward (f) and reverse (r) scan direction (light and dark colors); the $\mathrm{J}(\mathrm{V})$ curves obtained from the outdoor experiment (dash line) are collected only in reverse scan. The legend refers to the irradiance. The temperature of the device in the outdoor experiment is: $33.9{ }^{\circ} \mathrm{C}$ at $30 \mathrm{~mW} / \mathrm{cm}^{2} ; 43.3{ }^{\circ} \mathrm{C}$ at 70 $\mathrm{mW} / \mathrm{cm}^{2}$; and $46.5^{\circ} \mathrm{C}$ at $90 \mathrm{~mW} / \mathrm{cm}^{2}$.

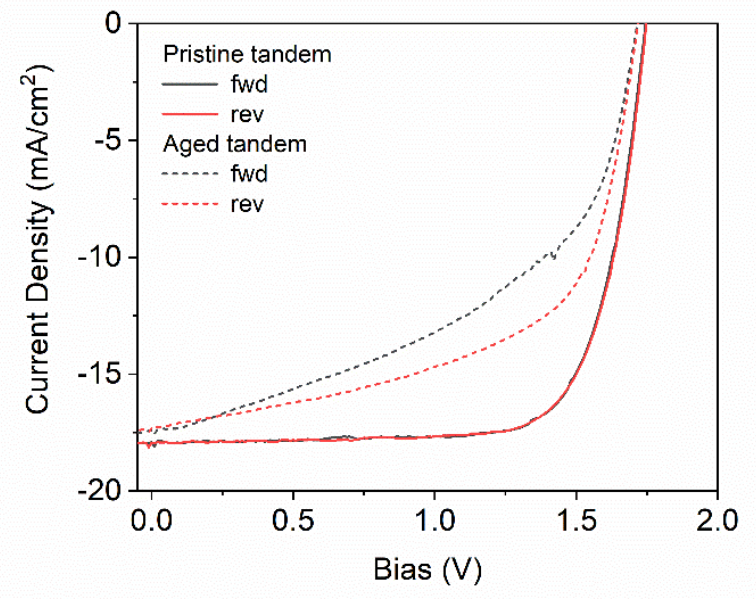

Figure S10. Hysteresis before and after the test-field experiment. Before the test-field experiment, the tandem shows negligible hysteresis (full line). Conversely, after the six-month 
experiment (dashed line), the tandem shows a strong hysteresis when the voltage scan direction changes from forward ( $J_{\mathrm{sc}}$ to $V_{\mathrm{oc}}$, black line) to reverse ( $V_{\mathrm{oc}}$ to $J_{\mathrm{sc}}$, red line).
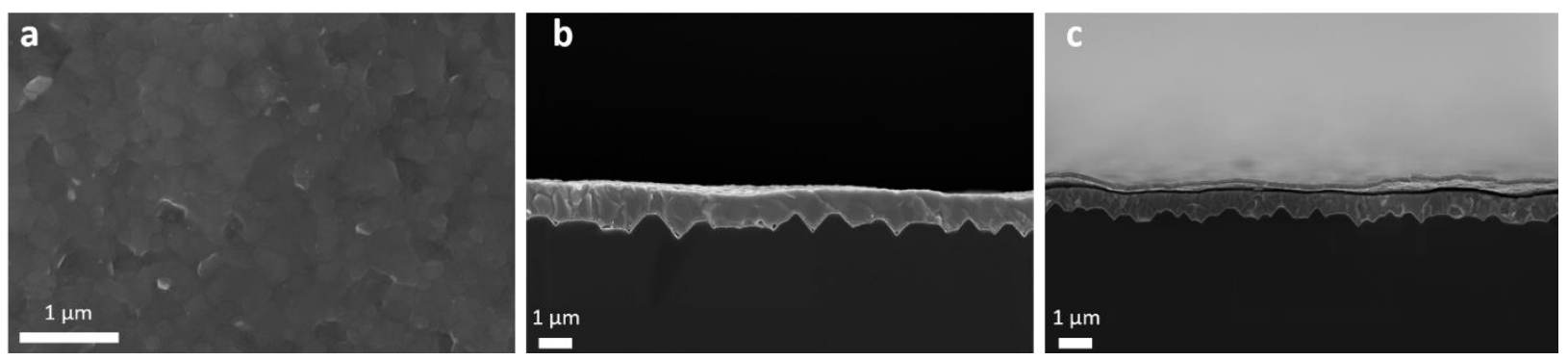

Figure S11. Scanning Electron Microscopy images of aged and fresh perovskite layer. (a) Top-view of the IZO layer under the silver busbar after six months of test-field experiments. The film shows the perovskite morphology with typical grain sizes. (b) Cross-section of the perovskite layer outside the active area after six months of test-field experiments. (c) Cross-section of the perovskite layer in the active area of a freshly prepared tandem.
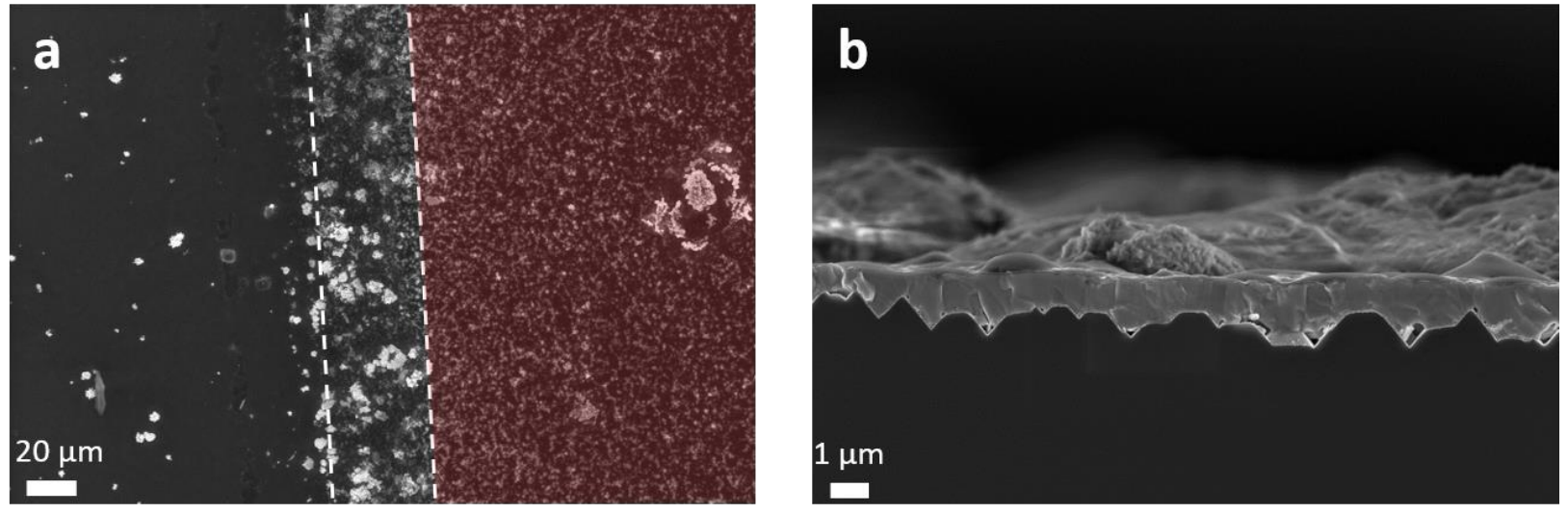

Figure S12. Scanning Electron Microscopy images of the silver electrode. (a) Top-view of the silver electrode busbar. The red area represent the silver contact, the area within the dashed lines the interface with the $\mathrm{MgF}_{2}$. (b) Cross-section of the silver electrode, focusing a cluster of $\operatorname{AgI}$. 


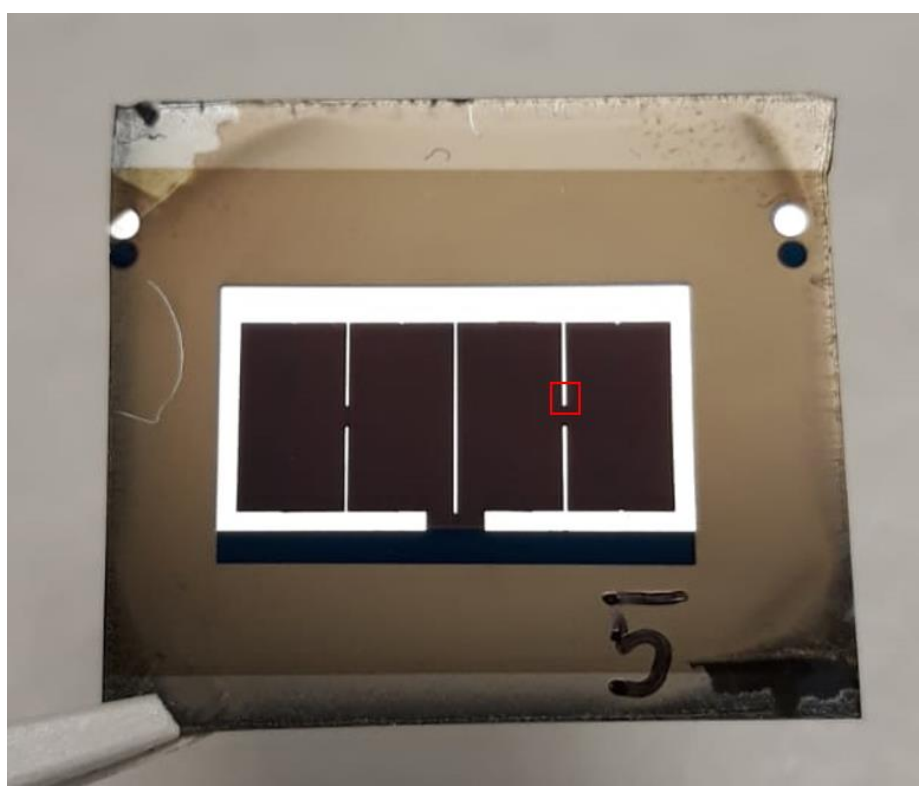

Figure S13. Position of the lamella for the TED-EDX analysis. The cross-section analysis was performed where the red square is located. The device represented in the picture is an illustration copy of the device used for the TEM analysis.

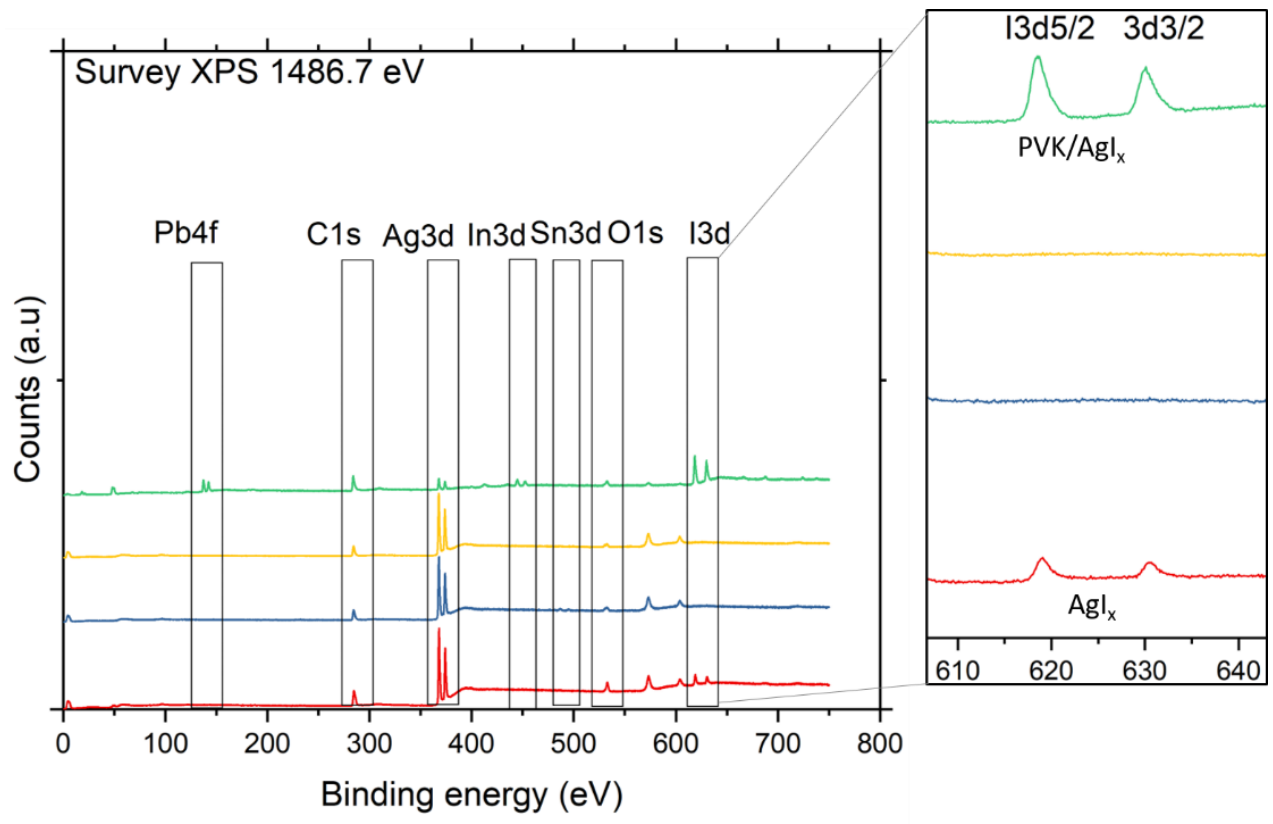

Figure S14. X-ray Photoelectron Spectroscopy analysis of the silver electrode. After the testfield experiment, the silver electrode of sample B was measured in four different spots: the silver 
paste used to tab the contact (yellow), the top of the silver busbar (blue), the area under the peeled electrode (green), and the bottom of the silver busbar forming the intimate contact with the evaporated silver (red).

a

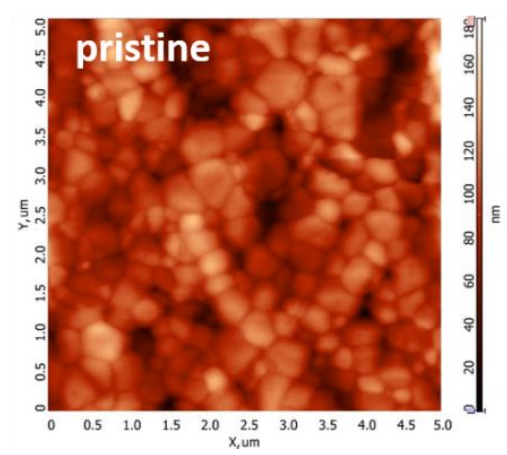

b

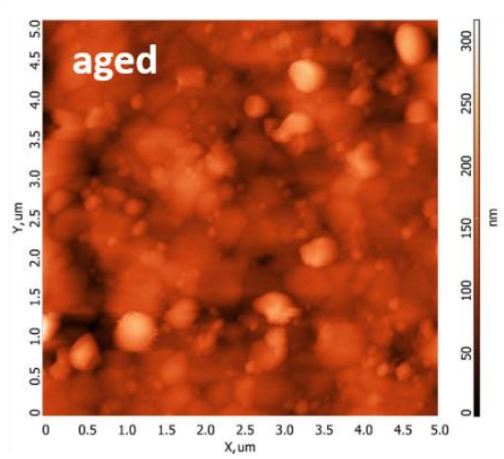

Figure S15. Atomic Force Microscopy of $\mathrm{C}_{60}$ on the perovskite film on a pristine and aged samples. The two samples are simultaneously fabricated on identical substrates mimicking the tandem's layout, but excluding the buffer layer (SnOx) and the TCO (IZO) to allow the morphological characterization. The pristine sample (a) is measured immediately, while the aged sample (b) is placed encapsulated similarly to the tandem for three weeks in the test-field before the measure. To enhance the effect of $\mathrm{C}_{60}$ aggregation, the thickness of the evaporated fullerene is increased from 20 to $40 \mathrm{~nm}$.

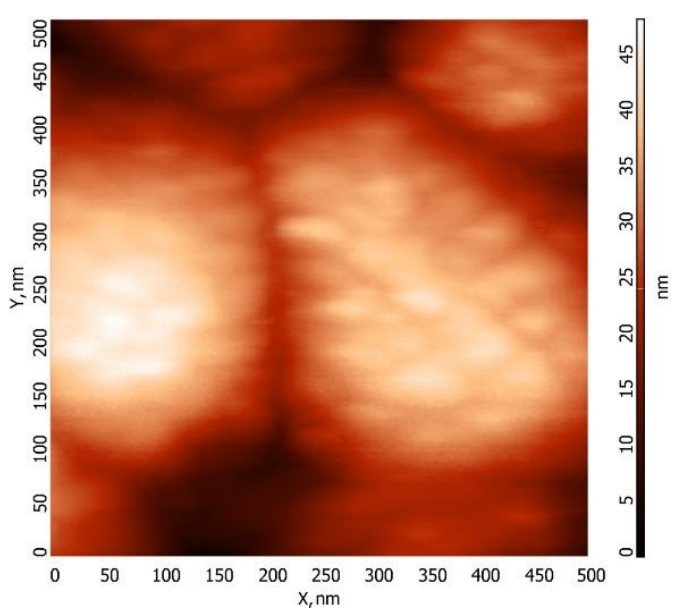


Figure S16. High-resolution Atomic Force Microscopy of $\mathbf{C}_{60}$ on the perovskite film. High resolution AFM topography of a perovskite grain covered by $40 \mathrm{~nm}$ of evaporated $\mathrm{C}_{60}$.

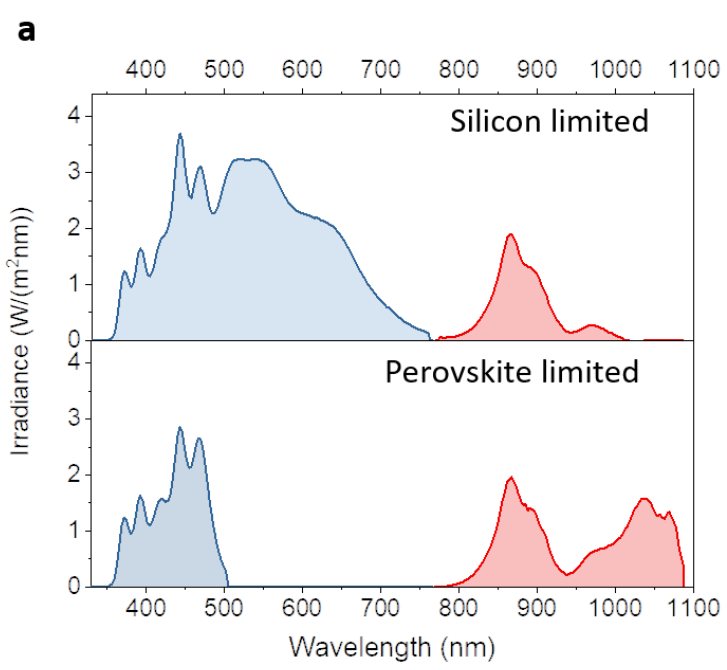

b

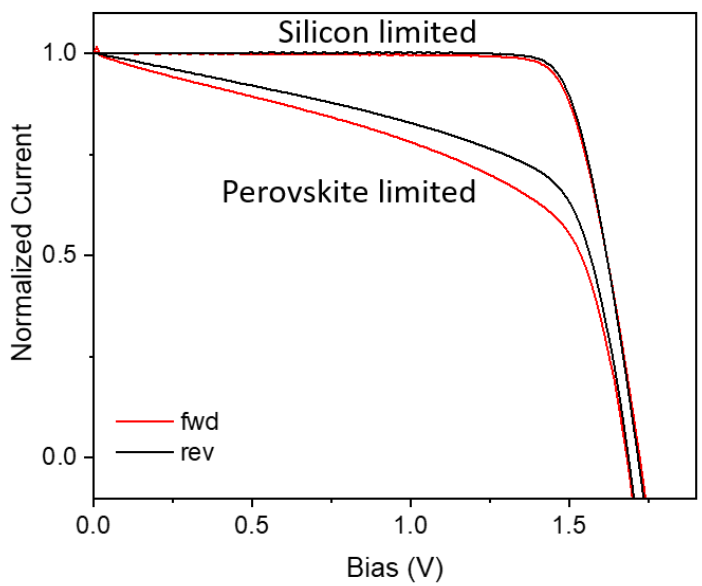

Figure S17. Sub-cell performances of Sample A. To isolate the contribution of each sub-cell we modified the irradiation spectrum of our LED-based solar simulator: excess of visible light to evaluate the silicon bottom-cell and excess of IR light to evaluate the perovskite top-cell. (a) The two spectra used and (b) the respective $\mathrm{J}(\mathrm{V})$ curves.

\section{REFERENCES}

1 Tress, W. et al. Performance of perovskite solar cells under simulated temperature-illumination real-world operating conditions. Nature Energy 4, 568-574 (2019). 